\title{
Urinary tract infection by a rare pathogen Cedecea neteri in a pregnant female with Polyhydramnios: rare case report from UAE
}

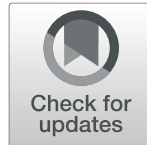

\author{
Hafiz Ahmad ${ }^{1,2^{*}}$, Talat Masroor ${ }^{3}$, Satyam A. Parmar ${ }^{2}$ and Debadatta Panigrahi ${ }^{4}$
}

\begin{abstract}
Background: Cedecea neteri is a gram-negative, oxidase-negative bacillus, a rare pathogen. Few reports are emerging globally about its antimicrobial resistance pattern especially in immunocompromised individuals with comorbidities.

Case presentation: In this paper, we report the first case of $C$. neteri causing urinary tract infection in a pregnant woman at a specialty care hospital in the Northern Emirates of Ras al Khaimah, UAE.

Discussion and conclusion: C. neteri is a rare and unusual pathogen, unlike routine gram-negative urinary tract pathogens from the family of Enterobacteriaceae and therefore may be missed or misidentified by routine laboratories using conventional microbiology identification techniques. Hence, Cedecea infections may be underreported. Physicians and microbiology technicians must be aware of such a rare pathogen, as most of the isolates are multi-drug-resistant and require combined antibiotic treatment with beta-lactamase inhibitors and hence pose a treatment challenge especially in immunocompromised patients with comorbidities. In recent years, it has been reported as an emerging opportunistic pathogen.
\end{abstract}

Keywords: Cedecea neteri, Rare pathogen, Urinary tract infection, Multi-drug resistance

\section{Background}

Cedecea neteri is a gram-negative, oxidase-negative bacillus - a rare pathogen with increasing case reports describing infections by the genus Cedecea. However, there have been very few cases of $C$. neteri reported so far, and none from urinary tract infection (UTI). In this paper, we report the first case to the best of our knowledge of C. neteri from a 27-year-old, young pregnant female with 35 weeks' gestation presenting with polyhydramnios.

Cedecea constitutes a rare pathogen of increasing importance. Cedecea species are known to have antibiotic

\footnotetext{
* Correspondence: hafeezaiims@gmail.com

'Department of Medical Microbiology \& Immunology, RAK College of Medical Sciences, RAK Medical and Health Sciences University, Ras al Khaimah, United Arab Emirates

${ }^{2}$ Clinical Laboratory, RAK hospital, Ras al Khaimah, UAE

Full list of author information is available at the end of the article
}

resistance genes and therefore difficult to treat infections caused by them, due to their broad-spectrum antibiotic resistance. There is emerging literature with case reports of bacteremia caused by $C$. neteri and more cases reported by other Cedecea species: C. davisae and C.lapagei. Nevertheless, the classical case of UTI by $C$. neteri species is rare. Literature review showing case of urinary catheter colonization by multidrug-resistant $C$. neteri in an elderly 88-year-old immunocompromised patient presenting with cellulitis, benign prostatic hyperplasia, and prolong use of Foleys catheter. The C.neteri isolated from urinary catheter was sensitive to most 2nd and 3rd generation cephalosporins and resistant to ampicillin along with $\beta$-lactamase inhibitor (Sulbactam) combination, suggesting AmpC $\beta$-lactamase production with the presence of multiple metallo $\beta$-lactamase genes [1]. In another report from Turkey, described UTI caused by

(c) The Author(s). 2021 Open Access This article is licensed under a Creative Commons Attribution 4.0 International License, which permits use, sharing, adaptation, distribution and reproduction in any medium or format, as long as you give appropriate credit to the original author(s) and the source, provide a link to the Creative Commons licence, and indicate if changes were made. The images or other third party material in this article are included in the article's Creative Commons licence, unless indicated otherwise in a credit line to the material. If material is not included in the article's Creative Commons licence and your intended use is not permitted by statutory regulation or exceeds the permitted use, you will need to obtain permission directly from the copyright holder. To view a copy of this licence, visit http://creativecommons.org/licenses/by/4.0/ The Creative Commons Public Domain Dedication waiver (http://creativecommons.org/publicdomain/zero/1.0/) applies to the data made available in this article, unless otherwise stated in a credit line to the data. 
Cedecea lapagei in a 40-year male with invasive brain surgery after 3 weeks of rehabilitation and was successfully treated with Ciprofloxacin [2]. Furthermore, there have been emerging reports of the association of $C$. neteri with other clinical presentations. To mention a few, a report of bacteremia in patients of heart disease [3], in patients with systemic lupus erythematosus [4], and a case report from Saudi Arabia where C. neteri was isolated from peritoneal fluid of an immunocompromised preterm neonate following intestinal perforation due to necrotizing enterocolitis [5]. Infections from Cedecea are mostly reported from immunocompromised patients with multiple comorbidities or invasive procedures, suggesting its role as an emerging opportunistic multi-drug resistant pathogen $[1,3,5]$.

\section{Case presentation}

Our patient was a 27-year-old pregnant woman at 35 weeks' gestation who visited the Gynecology outpatient department of RAK hospital with complaints of increased frequency, urgency, and dysuria; classical presentation of UTI. The patient was on follow-up for polyhydramnios. On examination, she was afebrile, well oriented to time, place, and person, with a pulse rate of $86 / \mathrm{min}$, blood pressure of $152 / 90 \mathrm{mmHg}$, a known hypertensive. However, there were no signs of cystitis or pyelonephritis based on abdominal and pelvis ultrasound. Her history revealed that she was multiparous and her first pregnancy ended with twins born by caesarian. During the second pregnancy, she developed polyhydramnios and was on continuous follow-up. Her mid-stream first-morning urine sample macroscopically was cloudy with yellow coloration. Microscopically it showed significant polymorph nuclear cells (18-20 cells/ high power field), no RBCs, and 3+ leukocyte esterase activity with plenty of leukocytes and bacteria with $<1$ squamous epithelial cell. Culture and identification yielded significant bacteriuria of $10^{5} \mathrm{CFU} / \mathrm{ml}$ of a gramnegative bacterium grown in Cysteine Lactose Electrolyte Deficient (CLED) agar. On further characterization, the isolate was identified by Phoenix automated microbial identification system (BD Diagnostics, Spars, Maryland), belonging to genus Cedecea and species neteri. To confirm the diagnosis of UTI, two mid-stream urine samples on consecutive days were further cultured. Both samples showed significant growth $\left(10^{5} \mathrm{CFU} / \mathrm{ml}\right)$ of $C$. neteri and thus confirmed the diagnosis.

Antimicrobial susceptibility testing showed the isolate to be resistant to Amikacin, Aztreonam, Cephalothin, Ceftriaxone, Ertapenem, Cefepime, Cefoxitin and susceptible to Ampicillin, Ampicloxacillin, Nitrofurantoin, Levofloxacin, Tigecycline \& Piperacillin/Tazobactum. The patient was treated with one gram of Augmentin (combination of 875 $\mathrm{mg}$ amoxicillin and $125 \mathrm{mg}$ clavulanic acid) for 5 days and urine culture was sterile post 1 week of treatment.

\section{Discussion and conclusion}

In females, UTI is most often caused by members of the family Enterobacteriaceae mainly $E$. coli, mostly endogenous, due to the proximity of the anus to the urethra and short urethra causing ascending infections in women. To the best of our knowledge, this is the first case of a UTI by a rare pathogen, Cedecea neteri, reported from the UAE, in a 35-week pregnant, multiparous female who has polyhydramnios and a known case of hypertension. Most likely $C$. neteri has been a colonic microbial flora of this patient.

Cedecea was described in 1981 as a separate genus of the Enterobacteriaceae family as it was phenotypically distinct from the other members of the family and named after $\mathrm{CDC}$, where the isolates were originally discovered and formerly called as CDC Enteric group 15 [6]. To date, six species of Cedecea have been reported with three species, Cedecea neteri, Cedecea lapagei, and Cedecea davisae, that are well characterized. C. neteri is named after the American physician-microbiologist Dr. Erwin Neter [7]. Cedecea species have not been reported to cause invasive infection in healthy individuals, but are considered opportunistic pathogens due to their isolation from severely immunocompromised patients. The case presented here is a pregnant mother who is immunocompromised and therefore more susceptible to infections. The growing fetus also exerts pressure on the urinary bladder, increasing residual urine, thereby affecting complete voiding and urine stasis predisposing to UTI.

These pathogens may be missed or misidentified by routine laboratories using conventional microbiology identification methods and hence may be underreported. Cedecea neteri strains are gram-negative, rodshaped, motile [8]. They are characterized by positive biochemical reactions for sucrose, D-sorbitol, and malonate fermentation tests $[3,9]$. Clinical history, laboratory diagnosis, and antimicrobial resistance pattern of Cedecea spp. resemblance to established pathogen Serratia spp. in terms of lipase positivity and resistance to cephalothin, and colistin (polymyxin E) [3, 7, 8]. C. neteri is distinguished from other Cedecea spp. with negative ornithine decarboxylase activity and its ability to ferment sucrose, D-sorbitol, and D-xylose [3].

Multidrug resistance in Cedecea spp. is attributed to the combination of AmpC production and porin deficiency in the cell wall $[8,10]$. However, no definite experimental evidence on this theory is available to this day. Cedecea clinical isolates display variable resistance patterns to major beta-lactam like penicillins, cephalosporins, monobactam, and carbapenems. The latest literature review indicates the highest frequency of 
Cedecea spp. resistance to ampicillin (43\%), followed by cephalothin (35\%), cefoxitin (35\%), cefazolin (22\%), ceftazidime from a total of 23 isolates reported to date [11]. Carbapenems and 4th generation cephalosporin resistance may be exhibited in Cedecea isolates harboring metallo- $\beta$-lactamase. $C$. neteri poses another unique feature to enhance its pathogenicity by Quorum sensing activity that has been lately studied in $C$. neteri strain SSMF04 and can lead to enhanced biofilm formation as well as enhanced antimicrobial resistance [12]. Fortunately, low resistance to fluoroquinolones and no resistance to macrolides have been documented. It is also encouraging to note that most of the reported cases have successful treatment outcomes and an active antibiotic stewardship policy with proactive microbiology laboratory diagnosis and antibiotic sensitivity testing can improve clinical outcomes. C. neteri infections have been reported worldwide, with clinical cases occurring from U.S., Spain, Saudi Arabia, and the latest one from UAE (Table 1 . summarizes all the $C$. neteri cases reported to date).

As summarized in Table 2, most Cedecea infections are reported in immunocompromised patients with underlying medical conditions like uncontrolled diabetes mellitus, chronic kidney disease, liver transplantation, malignancies, chronic obstructive pulmonary disease, and few isolated cases from catheters and central lines, indicating its opportunistic potential and warranting careful attention among these groups of patients.

C. davisae infections have been reported from a broad clinical spectrum with bacteremia as a common clinical presentation. Thirteen cases have been reported to date from different clinical specimens including; 46\% from blood, 23\% from sputum, urine, cutaneous and oral ulcers, and scrotal abscess. It is also noteworthy that the majority of infections were among patients $>50$ years of age with co-morbid conditions.

Apart from the endogenous source of infections from the gut, Cedecea infections are also documented from environmental samples and aquatic habitats. A case report of a patient with minor leg ulcer infection and subsequent bacteremia (Dalmaga et al. 2008) raises concerns of $C$. davisae infection from lake water.

C. lapagei have been more frequently isolated with pneumonia and bloodstream infections and to date, a total of 11 cases have been reported from blood, sputum, tissue, wound, peritoneal fluid and most patients had comorbid conditions like acute leukemia, type II diabetes mellitus, pulmonary tuberculosis, liver cirrhosis, and chronic obstructive pulmonary disease. Pediatric infections involving Cedecea are largely associated with C.lapagei, suggesting its role as an uncommon cause of nosocomial pneumonia and sepsis in infants with a history of multiple antibiotic treatment regimens and prolonged hospitalization.

It is also noteworthy, that most of the Cedecea infections are resistant to quinolones (ciprofloxacin, Cefotaxime (third-generation cephalosporin), Imipenem, and Meropenem (carbapenems) and intravenous combination with $\beta$-lactamase inhibitors (Piperacillin and Tazobactam) making it a potential multidrug-resistant pathogen. Therefore, clinicians and diagnostic laboratories need to be aware of such rare pathogens that are often drug-resistant and require combined antimicrobial therapy. A complete

Table 1 Cases of Cedecae neteri reported to date

\begin{tabular}{|c|c|c|c|c|c|c|c|c|}
\hline $\begin{array}{l}\text { Study } \\
\text { year }\end{array}$ & $\begin{array}{l}\text { Patient } \\
\text { (age/sex, } \\
\text { location) }\end{array}$ & $\begin{array}{l}\text { Cedecea } \\
\text { spp. }\end{array}$ & Infection & Diagnosis & $\begin{array}{l}\text { Antibiotic Sensitivity/ } \\
\text { Treatment }\end{array}$ & Antibiotic Resistance & $\begin{array}{l}\text { Clinical } \\
\text { Outcome }\end{array}$ & Reference \\
\hline 2021 & $\begin{array}{l}27 / F \\
\text { UAE }\end{array}$ & C. neteri & $\begin{array}{l}\text { Urinary tract } \\
\text { Infection }\end{array}$ & $\begin{array}{l}\text { Polyhydramnios, } \\
\text { hypertension }\end{array}$ & $\begin{array}{l}\text { amoxicillin and } \\
\text { clavulanic acid }\end{array}$ & $\begin{array}{l}\text { Amikacin, Aztreonam, } \\
\text { Cephalothin, Ceftriaxone, } \\
\text { Ertapenem, Cefepime, } \\
\text { Cefoxitin. }\end{array}$ & $\begin{array}{l}\text { Successful } \\
\text { recovery }\end{array}$ & $\begin{array}{l}\text { Hafiz et al. } \\
2021\end{array}$ \\
\hline 2018 & $\begin{array}{l}88 / M \\
\text { USA }\end{array}$ & C. neteri & $\begin{array}{l}\text { Colonization of } \\
\text { the Urinary } \\
\text { catheter }\end{array}$ & $\begin{array}{l}\text { Cellulitis, with } \\
\text { hypertension, } \\
\text { chronic } \\
\text { kidney disease } \\
\text { and benign } \\
\text { prostatic } \\
\text { hyperplasia }\end{array}$ & $\begin{array}{l}\text { cefmandole, } \\
\text { ceftazidime, } \\
\text { ceftriaxone, cefepime, } \\
\text { aztreonam, } \\
\text { nitrofurantoin, } \\
\text { ciprofloxacin, TMP/SMX } \\
\text { Piperacillin/tazobactam, }\end{array}$ & $\begin{array}{l}\text { Ampicillin/sulbactam, } \\
\text { cefazolin, cefoxitin }\end{array}$ & $\begin{array}{l}\text { Successful } \\
\text { recovery }\end{array}$ & $\begin{array}{l}\text { Ginn et al. } \\
\text { [1] }\end{array}$ \\
\hline 2017 & $\begin{array}{l}\text { Neonate/M } \\
\text { Saudi Arabia }\end{array}$ & C. neteri & Peritonitis & $\begin{array}{l}\text { Perforation of } \\
\text { Intestine }\end{array}$ & Not reported & $\begin{array}{l}\text { Piperacillin/tazobactam } \\
\text { and gentamicin }\end{array}$ & $\begin{array}{l}\text { Successful } \\
\text { recovery }\end{array}$ & $\begin{array}{l}\text { Arishi } \\
\text { et al. [5] }\end{array}$ \\
\hline 1995 & $\begin{array}{l}27 / F \\
\text { Spain }\end{array}$ & C. neteri & Bacteremia & $\begin{array}{l}\text { systemic lupus } \\
\text { erthematosus }\end{array}$ & $\begin{array}{l}\text { Intravenous } \\
\text { vancomycin, } \\
\text { ceftazidime, gentamicin }\end{array}$ & $\begin{array}{l}\text { Amoxicillin, } \\
\text { cephalosporins, } \\
\text { amoxicillin and clavulanic } \\
\text { acid, aminoglycosides }\end{array}$ & Died & $\begin{array}{l}\text { Augileria } \\
\text { et al. [4] }\end{array}$ \\
\hline 1982 & $\begin{array}{l}62 / M \\
\text { USA }\end{array}$ & C. neteri & Bacteremia & $\begin{array}{l}\text { Cardiac and } \\
\text { valvular heart } \\
\text { disease }\end{array}$ & $\begin{array}{l}\text { Cefamandole, } \\
\text { chloramphenicol, } \\
\text { n }\end{array}$ & $\begin{array}{l}\text { Cefalothin, ampicillin, } \\
\text { colistin }\end{array}$ & $\begin{array}{l}\text { Successful } \\
\text { recovery }\end{array}$ & $\begin{array}{l}\text { Farmer } \\
\text { et al. [3] }\end{array}$ \\
\hline
\end{tabular}


Table 2 Summary of representative Cedecea spp. cases reported to date

\begin{tabular}{|c|c|c|c|c|c|c|c|c|}
\hline $\begin{array}{l}\text { Study } \\
\text { year }\end{array}$ & $\begin{array}{l}\text { Patient } \\
\text { (age /sex, } \\
\text { location) } \\
\end{array}$ & $\begin{array}{l}\text { Cedecea } \\
\text { spp. }\end{array}$ & Infection & Diagnosis & $\begin{array}{l}\text { Antibiotic } \\
\text { Resistance }\end{array}$ & Treatment & $\begin{array}{l}\text { Clinical } \\
\text { Outcome }\end{array}$ & Reference \\
\hline 2019 & $\begin{array}{l}\text { Neonate/ } \\
\text { India }\end{array}$ & $\begin{array}{l}\text { C. } \\
\text { lapagei }\end{array}$ & $\begin{array}{l}\text { Nosocomial } \\
\text { Pneumonia }\end{array}$ & Late preterm & $\begin{array}{l}\text { Meropenem, } \\
\text { Colisitin, amikacin, } \\
\text { ceftazidime }\end{array}$ & $\begin{array}{l}\text { Piperacillin/ } \\
\text { tazobactam }\end{array}$ & Recovered & $\begin{array}{l}\text { Ramaswamy } \\
\text { et al. } 2019 \\
{[13]}\end{array}$ \\
\hline 2019 & $\begin{array}{l}41 / F \\
\text { USA }\end{array}$ & $\begin{array}{l}\text { C. } \\
\text { davisae }\end{array}$ & Biliary sepsis & Minimal change disease & $\begin{array}{l}\text { Ampicillin, } \\
\text { ceftriaxone, } \\
\text { cefuroxime }\end{array}$ & $\begin{array}{l}\text { Ciprofloxacin, } \\
\text { metronidazole }\end{array}$ & Recovered & $\begin{array}{l}\text { Kanakadandi } \\
\text { et al. [10] }\end{array}$ \\
\hline 2018 & $\begin{array}{l}52 / \mathrm{M} \\
\text { Mexico }\end{array}$ & $\begin{array}{l}\text { C. } \\
\text { lapagei }\end{array}$ & $\begin{array}{l}\text { Soft tissue bullae } \\
\text { leading to septic } \\
\text { shock }\end{array}$ & $\begin{array}{l}\text { Liver cirrhosis, esophageal } \\
\text { varices, hypertension }\end{array}$ & $\begin{array}{l}\text { Ampicillin, } \\
\text { cefazolin, } \\
\text { imipenem; } \\
\text { ampicillin/ } \\
\text { sulbactam }\end{array}$ & $\begin{array}{l}\text { Intravenous } \\
\text { imipenem, } \\
\text { clindamycin }\end{array}$ & Died & $\begin{array}{l}\text { Chavez } \\
\text { Herrera et al. } \\
2018[14]\end{array}$ \\
\hline 2017 & $\begin{array}{l}\text { Neonate/M } \\
\text { Brazil }\end{array}$ & $\begin{array}{l}\text { C. } \\
\text { lapagei }\end{array}$ & $\begin{array}{l}\text { Ventilator- } \\
\text { associated } \\
\text { pneumonia, } \\
\text { sepsis }\end{array}$ & & Not reported & $\begin{array}{l}\text { Multiple } \\
\text { courses } \\
\text { of antibiotics } \\
\text { including } \\
\text { meropenem }\end{array}$ & Recovered & $\begin{array}{l}\text { Kury et al. } \\
{[15]}\end{array}$ \\
\hline 2017 & $\begin{array}{l}\text { Neonate/M } \\
\text { India }\end{array}$ & $\begin{array}{l}\text { C. } \\
\text { lapagei }\end{array}$ & Late-onset sepsis & Preterm & $\begin{array}{l}\text { Imipenem, } \\
\text { meropenem, } \\
\text { aztreonam, } \\
\text { ceftazidime, } \\
\text { cefotaxime, } \\
\text { cefoxitin, } \\
\text { cefepime, }\end{array}$ & $\begin{array}{l}\text { Ampicillin, } \\
\text { cefotaxime }\end{array}$ & Recovered & $\begin{array}{l}\text { Ahmad et al. } \\
{[16]}\end{array}$ \\
\hline 2016 & $\begin{array}{l}\text { Neonate/F } \\
\text { India }\end{array}$ & $\begin{array}{l}\text { C. } \\
\text { lapagei }\end{array}$ & Neonatal sepsis & Term infant & $\begin{array}{l}\text { Amoxicillin/ } \\
\text { clavulanic } \\
\text { acid, ceftazidime, } \\
\text { ceftriaxone, } \\
\text { gentamicin, } \\
\text { cefuroxime, } \\
\text { piperacillin/ } \\
\text { tazobactam, } \\
\text { TMP/SMX }\end{array}$ & $\begin{array}{l}\text { Ciprofloxacin, } \\
\text { amikacin }\end{array}$ & Recovered & $\begin{array}{l}\text { Islam et al. } \\
{[17]}\end{array}$ \\
\hline 2015 & $\begin{array}{l}50 / M \\
\text { India }\end{array}$ & $\begin{array}{l}\text { C. } \\
\text { lapagei }\end{array}$ & $\begin{array}{l}\text { Superinfection of } \\
\text { malignant oral } \\
\text { ulcer }\end{array}$ & $\begin{array}{l}\text { Squamous cell } \\
\text { carcinoma of the right } \\
\text { buccal mucosa }\end{array}$ & $\begin{array}{l}\text { Ampicillin/ } \\
\text { sulbactam, } \\
\text { tetracycline, } \\
\text { tigecycline }\end{array}$ & Ciprofloxacin, & Recovered & $\begin{array}{l}\text { Biswal et al. } \\
{[18]}\end{array}$ \\
\hline 2006 & $\begin{array}{l}55 / M \\
\text { USA }\end{array}$ & $\begin{array}{l}\text { C. } \\
\text { lapagei }\end{array}$ & $\begin{array}{l}\text { CAPD-related } \\
\text { peritonitis }\end{array}$ & $\begin{array}{l}\text { CAPD, Hypertension, Liver } \\
\text { transplantation, cirrhosis, end- } \\
\text { stage renal disease }\end{array}$ & Not reported & $\begin{array}{l}\text { Initially } \\
\text { intravenous } \\
\text { vancomycin \& } \\
\text { gentamicin, } \\
\text { followed by } \\
\text { ceftazidime \& } \\
\text { gentamicin } \\
\text { added } \\
\text { to PD }\end{array}$ & Recovered & $\begin{array}{l}\text { Davis \& wall } \\
\text { [19] }\end{array}$ \\
\hline 2012 & $\begin{array}{l}\text { 54/M } \\
\text { Greece }\end{array}$ & $\begin{array}{l}\text { C. } \\
\text { davisae }\end{array}$ & Bacteremia & $\begin{array}{l}\text { Stage IV sigmoid } \\
\text { colon carcinoma }\end{array}$ & Tobramycin & Gentamicin & Recovered & $\begin{array}{l}\text { Akinosoglou } \\
\text { et al. [20] }\end{array}$ \\
\hline 2012 & $\begin{array}{l}\text { 20/M } \\
\text { USA }\end{array}$ & $\begin{array}{l}\text { C. } \\
\text { davisae }\end{array}$ & $\begin{array}{l}\text { Polymicrobial* } \\
\text { HCA pneumonia }\end{array}$ & Cystic Fibrosis & $\begin{array}{l}\text { All tested } \beta \text { - } \\
\text { lactams, } \\
\text { aminoglycosides, } \\
\text { fluoroquinolones, } \\
\text { tigecycline }\end{array}$ & TMP/SMX & Recovered & $\begin{array}{l}\text { Ismael et al. } \\
{[21]}\end{array}$ \\
\hline 2011 & $\begin{array}{l}\text { 52.M } \\
\text { USA }\end{array}$ & $\begin{array}{l}\text { C. } \\
\text { davisae }\end{array}$ & $\begin{array}{l}\text { Central line- } \\
\text { related } \\
\text { bacteremia }\end{array}$ & $\begin{array}{l}\text { Acute myeloid } \\
\text { leukemia, neutropenia, } \\
\text { C. difficile colitis }\end{array}$ & $\begin{array}{l}\text { Ceftazidime, } \\
\text { ciprofloxacin, } \\
\text { piperacillin/ } \\
\text { tazobactam }\end{array}$ & Imipenem & Recovered & $\begin{array}{l}\text { Abate et al. } \\
{[22]}\end{array}$ \\
\hline 2008 & $\begin{array}{l}\text { 67/M } \\
\text { Greece }\end{array}$ & $\begin{array}{l}\text { C. } \\
\text { davisae }\end{array}$ & $\begin{array}{l}\text { Leg ulcer, } \\
\text { bacteremeia }\end{array}$ & Uncomplicated DM & $\begin{array}{l}\text { Cephalothin } \\
\text { cefuroxime } \\
\text { sodium, } \\
\text { cefoxitin, }\end{array}$ & $\begin{array}{l}\text { Cefotaxime, } \\
\text { amikacin }\end{array}$ & Recovered & $\begin{array}{l}\text { Dalamaga } \\
\text { et al. [23] }\end{array}$ \\
\hline
\end{tabular}


Table 2 Summary of representative Cedecea spp. cases reported to date (Continued)

\begin{tabular}{|c|c|c|c|c|c|c|c|c|}
\hline $\begin{array}{l}\text { Study } \\
\text { year }\end{array}$ & $\begin{array}{l}\text { Patient } \\
\text { (age /sex, } \\
\text { location) }\end{array}$ & $\begin{array}{l}\text { Cedecea } \\
\text { spp. }\end{array}$ & Infection & Diagnosis & $\begin{array}{l}\text { Antibiotic } \\
\text { Resistance }\end{array}$ & Treatment & $\begin{array}{l}\text { Clinical } \\
\text { Outcome }\end{array}$ & Reference \\
\hline & & & & & $\begin{array}{l}\text { ampicillin, } \\
\text { piperacillin, } \\
\text { nitrofurantoin, } \\
\text { tetracycline }\end{array}$ & & & \\
\hline 2008 & $\begin{array}{l}\text { 47/M } \\
\text { Greece }\end{array}$ & $\begin{array}{l}\text { C. } \\
\text { lapagei }\end{array}$ & $\begin{array}{l}\text { Bacteremia and } \\
\text { wound infection } \\
\text { from cement- } \\
\text { related } \\
\text { chemical burns }\end{array}$ & Diabetes mellitus & $\begin{array}{l}\text { Gentamicin, } \\
\text { Tobramycin, } \\
\text { cephalothin, } \\
\text { cefoxitin, sodium, } \\
\text { cefoxitin, } \\
\text { ampicillin, } \\
\text { piperacillin, } \\
\text { nitrofurantoin, } \\
\text { tetracycline }\end{array}$ & $\begin{array}{l}\text { Cefotaxime, } \\
\text { amikacin }\end{array}$ & Recovered & $\begin{array}{l}\text { Dalamaga } \\
\text { et al. [24] }\end{array}$ \\
\hline 1983 & $\begin{array}{l}50 / M \\
\text { USA }\end{array}$ & $\begin{array}{l}\text { C. } \\
\text { davisae }\end{array}$ & Scrotal Abscess & $\begin{array}{l}\text { Chronic hypertensive } \\
\text { cardiac disease, } \\
\text { alcoholic hepatitis }\end{array}$ & $\begin{array}{l}\text { Ampicillin, } \\
\text { cephalothin, } \\
\text { cefamandole, } \\
\text { cefoxitin, } \\
\text { chloramphenicol }\end{array}$ & Tetracycline & Recovered & $\begin{array}{l}\text { Bae \& Sureka } \\
{[25]}\end{array}$ \\
\hline
\end{tabular}

antimicrobial susceptibility testing profile, especially with beta-lactam inhibitors is warranted to provide the best treatment options, especially among immunocompromised patients with comorbidities. (Tables 1,2 ) The present reported $C$. neteri isolate was resistant to most of the third and fourth generation cephalosporin except a few amino and carboxypenicillins that provided a successful treatment outcome. This report further adds to the existing literature on diagnosis, clinical presentation, antibiotic susceptibility, and clinical management of $C$. neteri infection.

\section{Abbreviations}

RAK: Ras al Khaimah; UTI: Urinary Tract Infections (UTI); CLED Agar: Cysteine Lactose Electrolyte Deficient Agar; CFU: Colony Forming Unit; CDC: Centers for Disease Control and Prevention; U.A.E: United Arab Emirates

\section{Acknowledgments}

The author's acknowledge Hospital CEO's - Dr. Raza Siddiqui and Dr. Jean Marc Gauer and RAK Medical \& Health Sciences University Dean Dr. KL Bairy and President Dr. S. Gurumadhva Rao for their support to publish the study.

\section{Authors' contributions}

HA: Made substantial contributions from drafting, conception, sample testing, and analysis and writing the Manuscript. TM- Made substantial contributions by managing the patient and providing clinical data and patient follow-up. SAP - Made substantial contributions to the laboratory analysis of the samples obtained. DP-Made substantial contributions to the interpretation of results and manuscript writing and proofreading the revised manuscripts. All authors have read and approved the manuscript.

\section{Funding}

Non-funded.

\section{Availability of data and materials}

All patient data is coded and kept confidential under authorization control in the hospital's Laboratory Information Management System and cannot be shared. However, parts of Cedecea neteri report generated by automated microbiology system can be shared upon request without compromising patient information. The data is user restricted and was accessed by the study PI- Dr. Hafiz Ahmad who is also a clinical microbiologist at the Department of Microbiology at RAK Hospital, Ras al Khaimah, UAE.

\section{Declarations}

\section{Ethics approval and consent to participate}

Ethical clearance and approval for the study were obtained from the RAK Medical and Health Sciences university ethical committee, approval letter no: RAKMHSU-REC-055-2019-F-M.

\section{Consent for publication}

Written and verbal patients consent for clinical sample testing, procedures, and publication for the academic purpose was taken by treating Gynecologist Dr. Talat Masroor at RAK hospital and is recorded electronically in LIMS RAK hospital software.

\section{Competing interests}

The authors declare that they have no competing interests.

\section{Author details}

'Department of Medical Microbiology \& Immunology, RAK College of Medical Sciences, RAK Medical and Health Sciences University, Ras al Khaimah, United Arab Emirates. ${ }^{2}$ Clinical Laboratory, RAK hospital, Ras al Khaimah, UAE. ${ }^{3}$ Department of Obstetrics \& Gynecology, RAK Hospital, Ras al Khaimah, UAE. ${ }^{4}$ College of Medicine, Ajman University, Ajman, UAE.

Received: 30 August 2020 Accepted: 9 June 2021

Published online: 02 July 2021

\section{References}

1. Ginn PS, Tart SB, Sharkady SM, Thompson DK. Urinary Catheter Colonization by Multidrug-Resistant Cedecea neteri in Patient with Benign Prostatic Hyperplasia. Case Rep Infect Dis. 2018:1-5. https://doi.org/10.1155/2018/752 0527.

2. Çekin Y, Kızılateş F, Dolu S, Öztoprak N, Cekin AH. The first urinary tract infection caused by Cedecea lapagei: a case report and review of the literature. Gazi Med J. 2014;20(2):193-5. https://doi.org/10.5455/GMJ-30-153282.

3. Farmer JJ, Sheth NK, Hudzinski JA, Rose HD, Asbury MF. Bacteremia due to Cedecea neteri sp. nov. J Clin Microbiol. 1982;16(4):775-8. https://doi.org/1 0.1128/jcm.16.4.775-778.1982

4. Aguilera A, Pascual J, Loza E, Lopez J, Garcia G, Liaño F, et al. Bacteremia with Cedecea neteri in a patient with systemic lupus erythematosus. Postgrad Med J. 1995;71(833):179-80. https:/doi.org/10.1136/pgmj.71.833.179.

5. Arishi HM, Daghriri AM, Gumairy FY, Ali YF. Cedecea neteri peritonitis as a complication of necrotizing Enterocolitis in a neonate. JCR. 2017;7:313-5. 
6. Janda JM, Abbott SL. Uncommon enterobacterial genera associated with clinical specimens. In: The Enterobacteria. Washington: ASM Press; 2006. p. 357-9. https://doi.org/10.1128/9781555817541.

7. Dalamaga M, Sotiropoulos GP, Vrioni G, Tsakris A. Cedecea: an "unknown" pathogen in the family of Enterobacteriaceae - its clinical importance, detection, and identification methods. Acta Microbiologica Hellenica [Internet]. 2014;59(1):17-28.

8. Ammenouche N, Dupont $\mathrm{H}$, Mammeri H. Characterization of a Novel AmpC -Lactamase Produced by a Carbapenem-Resistant Cedecea davisae Clinical Isolate. Antimicrob Agents Chemother. 2014;58(11):6942-5. https://doi.org/1 0.1128/AAC.03237-14.

9. Grimont PAD, Grimont F, Farmer JJ 3rd, Asbury MA. Cedecea davisae gen, nov.,ap. Nov. and Cedecae lapagei sp. Nov., new Enterobacteriaceae from clinical specimens. Int J Syst Bacteriol. 1981;31(3):317-26. https://doi.org/10.1 099/00207713-31-3-317.

10. Kanakadandi V, Sarao MS, Cunningham JM. A rare case of Cedecea davisae bacteremia presenting as biliary sepsis. Cureus. 2019;11(8):e5298. https://doi. org/10.7759/cureus.5298.

11. Thompson DK, Sharkady SM. Expanding Spectrum of opportunistic Cedecea infections: current clinical status and multidrug resistance. Int J Infect Dis. 2020;100:461-9. https://doi.org/10.1016/j.ijid.2020.09.036.

12. Tan KH, Tan JY, Yin WF, Chan KG. Genome analysis of quorum sensing Cedecea neteri SSMD04 leads to identification of its novel signaling synthase (cnel), cognate receptor (cneR) and an orphan receptor. PeerJ. 2015;3:e1216. https://doi.org/10.7717/peerj.1216.

13. Ramaswamy W, Gummadapu S, Suryanarayana N. Nosocomial pneumonia and sepsis caused by a rare organism Cedecea lapagei in an infant and a review of literature. BMJ Case Rep. 2019;12(7):e229854. https://doi.org/10.113 6/bcr-2019-229854

14. Chavez Herrera VR, Rosas De Silva MF, Orendain Alcaraz H, Ceja Espiritu G, Carrazco Peña K, Melnikov V. Death related to Cedecea lapagei in a soft tissue bullae infection: a case report. J Med Case Rep. 2018;12:328.

15. Kury CMH, Yabrudi AA, de Souza TB, de Souza EC, E Silva Costa LT, Soares $\mathrm{CB}$, et al. First reported case of ventilator-associated pneumonia and sepsis caused by Cedecea lapagei in a Brazilian neonatal intensive care unit. J Pediatr Infect Dis Soc. 2017;6(2):209-10. https://doi.org/10.1093/jpids/ piw077.

16. Ahmad N, Ali SM, Khan AU. First reported New Delhi metallo- $\beta$-lactamase-1producing Cedecea lapagei. Int J Antimicrob Agents. 2017;49(1):118-9. https://doi.org/10.1016/j.ijantimicag.2016.10.001.

17. Islam AKS, Bora R, Ahmed R, Borah AK, Ramasamy S. A case of neonatal sepsis with pneumonia due to Cedecea lapagei. IOSR-JDMS. 2016;15:84-5.

18. Biswal I, Hussain NA, Grover RK. Cedecea lapagei in a patient with malignancy: report of a rare case. J Cancer Res Ther. 2015;11:646.

19. Davis O, Wall BM. "Broom straw peritonitis" secondary to Cedecea lapagei in a liver transplant recipient. Perit Dial Int. 2006;26(4):512-3. https://doi.org/1 $0.1177 / 089686080602600422$

20. Akinosoglou K, Perperis A, Siagris D, Goutou P, Spiliopoulou I, Gogos CA, et al. Bacteraemia due to Cedecea davisae in a patient with sigmoid colon cancer: a case report and brief review of the literature. Diagn Microbiol Infect Dis. 2012; 74(3):303-6. https://doi.org/10.1016/j.diagmicrobio.2012.06.019.

21. Ismaael TG, Zamora EM, Khasawneh FA. Cedecea davisae's role in a polymicrobial lung infection in a cystic fibrosis patient. Case Rep Infect Dis. 2012:2012:176864.

22. Abate G, Qureshi S, Mazumder SA. Cedecea davisae bacteremia in a neutropenic patient with acute myeloid leukemia. J Inf Secur. 2011;63:83-5.

23. Dalamaga M, Karmaniolas K, Arsenis G, Pantelaki M, Daskalopoulou K, Papadavid $\mathrm{E}$, et al. Cedecea lapagei bacteremia following cement-related chemical burn injury. Burns. 2008a;34(8):1205-7. https://doi.org/10.1016/j. burns.2007.09.001.

24. Dalamaga M, Pantelaki M, Karmaniolas K, Matekovits A, Daskalopoulou K. Leg ulcer and bacteremia due to Cedecea davisae. Eur J Dermatol. 2008b; 18(2):204-5. https://doi.org/10.1684/ejd.2008.0374.

25. Bae BHC, Sureka SB. Cedecea davisae isolated from scrotal abscess. J Urol. 1983;130(1):148-9. https://doi.org/10.1016/50022-5347(17)51004-6.

\section{Publisher's Note}

Springer Nature remains neutral with regard to jurisdictional claims in published maps and institutional affiliations.

Ready to submit your research? Choose BMC and benefit from:

- fast, convenient online submission

- thorough peer review by experienced researchers in your field

- rapid publication on acceptance

- support for research data, including large and complex data types

- gold Open Access which fosters wider collaboration and increased citations

- maximum visibility for your research: over $100 \mathrm{M}$ website views per year

At BMC, research is always in progress.

Learn more biomedcentral.com/submissions 